\title{
A Note on Coherent States Related to Weighted Shifts
}

\author{
By \\ Włodzimierz MLAK* and Jan STOCHEL**
}

\$1. Let $\mathscr{H}$ be a separable complex Hilbert space with a fixed orthonormal basis $\left\{e_{n}\right\}_{n=0}^{\infty}$. Denote by $S$ a forward isometric shift such that $S e_{n}=e_{n+1}$ for $n \geq 0$ and by $D$ a diagonal operator determined by the equality $D e_{n}=w_{n} e_{n}$ for $n \geq 0$. Assume that $w_{n}>0$ for $n \geq 0$. The operator $W:=S D$ is called a weighted shift (with weights $w_{n}$ ). Notice that $W$ is an invertible and irreducible operator such that $\mathscr{D}(W)=\mathscr{D}(D)(\mathscr{D}(A)$ stands for the domain of an operator $A)$. Since $S$ is bounded and $D$ is self-adjoint, we have

$$
W^{*}=D S^{*} \text {. }
$$

Using (1.1) one can describe the adjoint $W^{*}$ of the operator $W$ as follows

$$
\mathscr{D}\left(W^{*}\right)=\left\{f \in H: \sum_{n=0}^{\infty}\left|\left(f, e_{n+1}\right)\right|^{2} w_{n}^{2}<+\infty\right\}
$$

and

$$
W^{*} f=\sum_{n=0}^{\infty}\left(f, e_{n+1}\right) w_{n} e_{n}, \quad f \in \mathscr{D}\left(W^{*}\right)
$$

Thus

$$
W^{*} e_{0}=0, W^{*} e_{n}=w_{n-1} e_{n-1}, \quad n>0 .
$$

The celebrated example of a weighted shift is the quantum creation operator $a^{+}$which acts as follows: $a^{+} e_{n}=\sqrt{n+1} e_{n+1}$ for $n \geq 0$. The basic functional model of $a^{+}$belongs to Bargmann [1]. Let us enter into details. Denote by $\mu$ the Borel measure on $C^{1}$ defined by $d \mu(z)=\frac{1}{\Pi} e^{-|z|^{2}} d m(z)$, where $m$ is the planar Lebesgue measure. It is proved in [1] that the set of all entire functions belonging to $L^{2}(\mu)$ forms a closed linear subspace of $L^{2}(\mu)$. We denote this subspace by $B^{2}$. The main result of $[1]$ states that:

Communicated by H. Araki, May 31, 1991.

1991 Mathematics Subject Classifications: 47B37, 47B20, 81R30

* Instytut Matematyczny PAN, św. Tomasza 30, 31-027 Kraków, Poland

*** Instytut Matematyki UJ. Reymonta 4, 30-059 Kraków, Poland 

$a^{+}$is unitarily equivalent to the operator $M$ of multiplication by the independent variable $z$ in $B^{2}$

and

$$
\begin{aligned}
& a:=\left(a^{+}\right)^{*} \text { is unitarily equivalent to the differential } \\
& \text { operator } \frac{d}{d z} \text { acting in } B^{2} \text { with } \mathscr{D}\left(\frac{d}{d z}\right)=\left\{f \in B^{2}: \frac{d}{d z} f \in B^{2}\right\} .
\end{aligned}
$$

Notice that the operator of multiplication by the independent variable $z$, defined in $L^{2}(\mu)$, is a normal extension of $M$. Thus $W$ and consequently $a^{+}$are unbounded subnormal operators.

Recall that $M^{*}=\frac{d}{d z}$, i.e. $\mathscr{D}\left(M^{*}\right)=\left\{f \in B^{2}: \frac{d}{d z} f \in B^{2}\right\}$ and $M^{*} f(z)=\frac{d}{d z} f(z)$ for $f \in \mathscr{D}\left(M^{*}\right)$. Define for $u \in C^{1}$ and $z \in C^{1}$ the function $h_{u}(z)=e^{u z}$. It is plain that $h_{u} \in B^{2}$ and, moreover, $\frac{d}{d z} h_{u}(z)=u h_{u}(z)$ for all $z \in C^{1}$. Thus $M^{*} h_{u}=u h_{u}$, which means that for every $u \in C^{1}$, the function $h_{u}$ is an eigenfunction of $M^{*}$ with the eigenvalue $u$. Hence the point spectrum of $M^{*}$ is equal to $C^{1}$. Consequently the point spectrum of the annihilation operator $a$ coincides with $C^{1}$.

The crucial point of our investigations is that the sequence $\left\{e_{n}\right\}_{n=0}^{\infty} \subset B^{2}$ defined by $e_{n}(z)=\frac{z^{n}}{\sqrt{n !}}(n \geq 0)$, is an orthonormal basis of $B^{2}$ such that $\left(M e_{n}\right)$ $(z)=z e_{n}(z)=\sqrt{n+1} e_{n+1}(z)$ for $n \geq 0$. Since $h_{u}(z)=\sum_{n=0}^{\infty} \frac{u^{n}}{\sqrt{n !}} \cdot \frac{z^{n}}{\sqrt{n !}}=\sum_{n=0}^{\infty} \frac{u^{n}}{\sqrt{n !}}$ $e_{n}(z)$ for all $z \in C$, one can show that for any $u \in C^{1}$, the series $\sum_{n=0}^{\infty} \frac{u^{n}}{\sqrt{n !}} e_{n}$ converges in $B^{2}$ to $h_{u}$. Setting $w_{n}=\sqrt{n+1}$ for $n \geq 0$, we can write

$$
h_{u}=e_{0}+\sum_{n=1}^{\infty} \frac{u^{n}}{w_{0} \cdot \ldots \cdot w_{n-1}} e_{n}, \quad u \in C^{1}
$$

In particular we have $\left\|h_{u}\right\|^{2}=e^{|u|^{2}}$ for $u \in C^{1}$. Thus the function $e^{-|u|^{2} / 2} h_{u} \in B^{2}$ is a normalized eigenfunction of the annihilation operator $a$. It is called a coherent normalized quantum state of the electromagnetic field; such states are used in the quantum optics (see [3] and [5]).

\$2. Let $W$ be a weighted shift with positive weights $w_{n}$, i.e. $W e_{n}=w_{n} e_{n+1}$ and $w_{n}>0$ for $n \geq 0$. The equality (1.7) suggests that the proper candidate for a coherent state related to $W$ would be the orthogonal series

$$
h(z)=e_{0}+\sum_{n=1}^{\infty} \frac{z^{n}}{w_{0} \cdot \ldots \cdot w_{n-1}} e_{n}
$$

In order to have a nonempty region of convergence of the series defining $h(z)$, we assume, following the Cauchy-Hadamard theorem, that 


$$
r(W)=\lim \inf _{n \rightarrow x}\left(w_{0} \cdot \ldots \cdot w_{n-1}\right)^{1 / n}>0 .
$$

Denote by $S(W)$ the open disc $\left\{z \in C^{1}:|z|<r(W)\right\}$. Call $r(W)$ and $S(W)$ the coherence radius of $W$ and the coherence spectrum of $W$, respectively.

Let $z$ be an arbitrary element of $S(W)$. Take a real number $r_{0}$ such that $|z|<r_{0}<r(W)$. Then there exists $n_{0}>0$ and $q$ such that $0<q<1$ and

$$
\frac{r_{0}}{\left(w_{0} \cdot \ldots \cdot w_{n-1}\right)^{1 / n}} \leq q, \quad n \geq n_{0} .
$$

Therefore

$$
\frac{|z|^{2 n}}{\left(w_{0} \cdot \ldots \cdot w_{n-1}\right)^{2}} \leq q^{2 n}, \quad n \geq n_{0} .
$$

This in turn implies that the orthogonal series defining $h(z)$ is convergent in $H$. It follows from (2.1) that

$$
\left(h(z), e_{0}\right)=1
$$

and

$$
\left(h(z), e_{n+1}\right)=\frac{z^{n+1}}{w_{0} \cdot \ldots \cdot w_{n}}, \quad n \geq 0 .
$$

Thus

$$
\left|\left(h(z), e_{n+1}\right)\right|^{2} w_{n}^{2}=\frac{|z|^{2(n+1)}}{\left(w_{0} \cdot \ldots \cdot w_{n-1}\right)^{2}} \quad n \geq 1 .
$$

One can deduce from (2.4) and (2.7) that

$$
\sum_{n=0}^{\infty}\left|\left(h(z), e_{n+1}\right)\right|^{2} w_{n}^{2}<+\infty,
$$

which shows, by (1.2), that

$$
h(z) \in \mathscr{D}\left(W^{*}\right) .
$$

Consequently, by (1.3) and (2.6), we get

$$
W^{*} h(z)=z e_{0}+\sum_{n=1}^{\infty} \frac{z^{n+1}}{w_{0} \cdot \ldots \cdot w_{n-1}} e_{n}=z h(z) .
$$

Arguing similarly to the above, one can show that for any $z \in S(W)$, the dimension of the kernel of $z I-W^{*}$ is equal to 1 (see [6] for the bounded case). Summing up we have proved the following

Theorem 2.1. If $W$ is a weighted shift with positive weights and $r(W)>0$, then 


$$
\begin{array}{cc}
h(z) \in \mathscr{D}\left(W^{*}\right), & z \in S(W), \\
W^{*} h(z)=z h(z), & z \in S(W)
\end{array}
$$

and

$$
\operatorname{dim} \operatorname{Ker}\left(z I-W^{*}\right)=1 . \quad z \in S(W) .
$$

In the sequel we call $h(z)$ the coherent state of $W$ at $z \in S(W)$.

Consider again the creation operator $W=a^{+}$, i.e. $W e_{n}=\sqrt{n+1} e_{n+1}$ for $n \geq 0$. Then one can easy check that $r(W)=+\infty$ and $S(W)=\mathbb{C}^{1}$. Moreover, the corresponding coherence state $h(z)$ is expressed by the formula

$$
h(z)=\sum_{n=0}^{\infty} \frac{z^{n}}{\sqrt{n !}} e_{n}, \quad z \in \mathbb{C}^{1}
$$

and $a h(z)=z h(z)$ for $z \in C^{1}$, i.e. $h(z)$ is an eigenfunction of the annihilation operator $a$. Notice that this result has been obtained without appealing to the Bargmann analytic model of $a^{+}$(see Section 1). However this model has inspired all we have presented in Section 2.

3. This section deals with subnormal weighted shifts. Let $W$ be a weighted shift within the space $H$. Assume that $W$ is a subnormal operator, i.e. there is a superspace $K$ of $H$ and a normal operator $M$ within $K$ such that $W \subset M$ ( $M$ will be called a normal extension of $W$ ). It is easy to see that $W$ has the following properties

$$
P \mathscr{D}(M) \subset \mathscr{D}\left(W^{*}\right)
$$

and

$$
W^{*} P f=P M^{*} f, \quad f \in \mathscr{D}(M),
$$

where $P$ is the orthogonal projection of $K$ onto $H$. In particular we have $\left\|W^{*} e_{n+1}\right\| \leq\left\|M^{*} e_{n+1}\right\|=\left\|M e_{n+1}\right\|=\left\|W e_{n+1}\right\|=w_{n+1}$ for $n \geq 0$. But $\left\|W^{*} e_{n+1}\right\|$ $=w_{n}$, so $w_{n} \leq w_{n+1}$ for $n \geq 0$. This in turn implies that

$$
r(W)=\lim _{n \rightarrow \infty} w_{n} \text {. }
$$

If $W$ is unbounded, then, in virtue of (3.3), we have

$$
r(W)=+\infty \text { and } S(W)=C^{1} .
$$

On the other hand, if $W$ is bounded, then

$$
r(W)=\lim _{n \rightarrow x} w_{n}=\|D\|=\|W\| .
$$

Let $M$ be a normal extension of the weighted shift $W$ with positive weights $w_{n}$. Let $E$ be the spectral measure of $M$. Denote by $K_{0}$ the closed linear span of the vectors $E(\sigma) e_{0}$, where $\sigma$ runs through the plane Borel sets. Since 


$$
e_{n}=\left(w_{0} \cdot \ldots \cdot w_{n-1}\right)^{-1} W^{n} e_{0}=\left(w_{0} \cdot \ldots \cdot w_{n-1}\right)^{-1} M^{n} e_{0}, \quad n \geq 1,
$$

we get $e_{n} \in K_{0}$ for $n \geq 0$. By the spectral theorem, the part of $M$ in $K_{0}$ is canonically isomorphic to the operator of multiplication by the independent variable $z$ in the space $L^{2}(\mu)$ with $\mu(\cdot)=\left(E(\cdot) e_{0}, e_{0}\right)$. The unitary isomorphism is uniquely determined by the correspondence $E(\sigma) e_{0} \rightarrow \chi_{\sigma}\left(\chi_{\sigma}\right.$ stands for the characteristic function of the set $\sigma$ ). Denote by $e_{n}(\cdot)$ the image (via the canonical isomorphism) of the vector $e_{n}$ in $L^{2}(\mu), n \geq 0$. Since $M e_{0}=W e_{0}=w_{0} e_{1}$ and $\left(M e_{0}\right)(z)=z$, we get $e_{1}(z)=\frac{z}{w_{0}}$. Similarly, the equality $M e_{1}=w_{1} e_{2}$ implies $z e_{1}(z)=w_{1} e_{2}(z)$, so $e_{2}(z)=\frac{z^{2}}{w_{0} w_{1}}$. Applying the induction procedure, we come to the conclusion

$$
e_{n}(z)=\frac{z^{n}}{w_{0} \cdot \ldots \cdot w_{n-1}}, \quad n \geq 1
$$

Thus we have described in an explicit form the orthonormal basis of the picture $B^{2}(\mu)$ of the space $H$ in $L^{2}(\mu)$. It is plain - due to $W \subset M-$ that $\left(\widehat{W} e_{n}(\cdot)\right)(z)=$ $z e_{n}(z)=w_{n} e_{n+1}(z)$ for $n \geq 0$, where $\widehat{W}$ is the picture of $W$ in $B^{2}(\mu)$. Hence $\widehat{W}$ is the functional model of $W$. Notice that in the above we have exploited merely the simplest form of the spectral theorem. For general theory of functional models for unbounded cyclic operators we refer the reader to [7].

Let us assume that the subnormal weighted shift $W$ satisfies the following condition:

$$
\mu\left(C^{1} \backslash S(W)\right)=0 .
$$

Given $f \in H$, we define the function $\widehat{f}: S(W) \rightarrow C$ by $\widehat{f}(z)=(f, h(\bar{z}))$ for $z \in S(W)$. It follows from (2.1) and (3.6) that

$$
\widehat{f}(z)=\sum_{n=0}^{\infty}\left(f, e_{n}\right) e_{n}(z), \quad z \in S(W), f \in H .
$$

Notice that $\widehat{f}$ is analytic in $S(W)$. Since $\|f\|^{2}=\sum_{n=0}^{\infty}\left|\left(f, e_{n}\right)\right|^{2}$, one can show, using (3.7), (3.8) and the Riesz-Fischer theorem that $\widehat{f} \in B^{2}(\mu)$. Moreover, by the Parseval equality, we have

$$
\int_{s(w)}|\widehat{f}(z)|^{2} d \mu(z)=\|f\|^{2}, \quad f \in H .
$$

An application of the polarization formula gives us

$$
(f, g)=\int_{s(w)} \widehat{f}(z) \overline{\widehat{g}(z)} d \mu(z), \quad f, g \in H .
$$

Summing up we have proved the following theorem. 
Theorem 3.1. Let $W$ be a subnormal weighted shift with positive weights. If $\mu\left(C^{1} \backslash S(W)\right)=0$, then the map $f \rightarrow \widehat{f}$ is a unitary isomorphism of $H$ onto $B^{2}(\mu)$.

Let us consider the Bergmann space $A^{2}$. Then the operator $W$ defined on $A^{2}$ by

$$
(W f)(z)=z f(z), \quad|z|<1, f \in A^{2},
$$

is a subnormal weighted shift with weights $w_{n}=\sqrt{\frac{n+1}{n+2}}$. In this particular case $S(W)=\left\{z \in C^{1}:|z|<1\right\}$ and $\mu=m=$ the planar Lebesgue measure.

$\$ 4$. Let $W$ be a subnormal weighted shift with corresponding space $B^{2}(\mu)$. Similarly to Section 3 we assume that $\mu\left(C^{1} \backslash S(W)\right)=0$. It follows from Theorem 3.1 that the map $f \rightarrow \widehat{f}$ sends $H$ onto the whole $B^{2}(\mu)$. Given $z \in S(W)$, we denote by $P_{z}$ the orthogonal projection of $H$ onto the one-dimensional space spanned by the vector $h(\bar{z})$. An elementary computation shows that

$$
P_{z} f=\frac{\widehat{f}(z)}{\|h(\bar{z})\|^{2}} h(\bar{z}), \quad f \in H, z \in S(W) .
$$

(Notice that (2.1) implies $\|h(\bar{z})\|^{2}=1+\sum_{n=1}^{\infty} \frac{|z|^{2 n}}{\left(w_{0} \cdot \ldots \cdot w_{n-1}\right)^{2}}>0$ for $z \in S(W)$ ).
It follows from (4.1) that

$$
\left(P_{z} f, g\right)=\left(P_{z} f, P_{z} g\right)=\widehat{f}(z) \overline{\widehat{g}(z)} \quad \frac{1}{\|h(\bar{z})\|^{2}}, \quad f, g \in H, z \in S(W) .
$$

Using (3.10) and (4.1), we get

$$
(f, g)=\int_{s(w)} \widehat{f}(z) \overline{\widehat{g}(z)} d \mu(z)=\int_{s(w)}\left(P_{z} f, g\right)\|h(\bar{z})\|^{2} d \mu(z), \quad f, g \in H .
$$

This can be rewritten in terms of the weak integral as follows

$$
f=\int_{s(w)} P_{:} f\|h(\bar{z})\|^{2} d \mu(z), \quad f \in H .
$$

Substituting (4.1) into (4.3) we get

$$
f=\int_{s(w)} \widehat{f}(z) h(\bar{z}) d \mu(z), \quad f \in H .
$$

This in turn implies that

$$
A f=\int_{s(w)} \widehat{f}(z) A h(\bar{z}) d \mu(z), \quad f \in H,
$$

for any bounded linear operator $A$ within $H$.

Summing up we have proved the following theorem. 
Theorem 4.1. Let $W$ be a subnormal weighted shift with positive weights. Assume that $\mu\left(C^{1} \backslash S(W)\right)=0$. Then

$$
I=\int_{s(w)} P_{:}\|h(\bar{z})\|^{2} d \mu(z), \quad \text { (weak integral), }
$$

where I stands for the identity operator on $H$. Moreover, if $A$ is a bounded linear operator on $H$, then the equality (4.5) holds.

Let us consider again the quantum creation operator $a^{+}$. It is well-known (cf. [1]) that in that case $B^{2}(\mu)$ coincides with the Bargmann space $B^{2}$ and $d \mu(z)=\frac{1}{\Pi} e^{-|=|^{2}} d m(z)$ ( $m$ is the planar Lebesgue measure). Moreover, by (1.7), we have $\|h(z)\|^{2}=e^{|z|^{2}}$ for $z \in C^{1}$. Since $S\left(a^{+}\right)=C^{1}$, the integral formula (4.6) turns into

$$
\frac{1}{\Pi} \int_{C} P_{z} d m(z)=I
$$

This is the celebrated Glauber-Klauder basic formula of quantum optics (cf. [3]).

\section{References}

[1] Bargmann, V., On a Hilbert space of analytic functions and associated integral transform, Comm. Pure Appl. Math., 19 (1961), 187-214.

[2] Berezin, F.A. and Shubin, M.A., Schrödinger equation, (Moscow, 1983).

[3] Klauder, J.R. and Sudarshan, E.C.G., Fundamentals of quantum optics, (New York, Amsterdam, 1968).

[4] Mlak, W., Notes on quantum circular operators, Part I, Preprint 303, Institute of Mathematics, Polish Academy of Sciences (1984).

[5] Perina, J., Quantum statistics of linear and nonlinear optical phenomena, (Reidel P. Comp., Boston, Lancaster, 1984).

[6] Shields, A.L., Weighted shifts and analytic function theory, Math. Surveys, 13, American Mathematical Society, (Providence, Rhode Island, 1974), 49-128.

[7] Stochel, J., and Szafraniec, F.H., On normal extensions of unbounded operators. III. Spectral properties, Publ. RIMS, Kyoto Univ.. 25 (1989), 105-139.

[8] Volkin, H.C., Phase operators, J. Math. Phys., 14 (1973), 1965-1976. 
